Recepción: 28 / 10 / 2016

Aceptación: 16 / 02 / 2017

Publicación: 29 / 04 / 2017

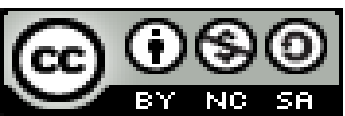

Ciencias económicas y empresariales

Artículo de investigación

\title{
Breve reseña acerca de la planeación estratégica
}

\author{
Brief overview of strategic planning
}

Breve visão geral de planejamento estratégico

Marcelo I. Ortiz-Díaz ${ }^{\mathrm{I}}$ marcelortizdiaz@gmail.com

Correspondencia: marcelortizdiaz@gmail.com

Magister en Administración y Marketing, Especialista en Gestión de Marketing, Diploma Superior en Gestión Administrativa, Licenciado en Ciencias Administrativas Administrador de Empresas, Ingeniero Comercial, Universidad Técnica de Ambato, Ambato, Ecuador. 


\section{Resumen}

La Planificación Estratégica sirve para determinar que somos (Misión) a donde queremos llegar (Visión) y que debemos que hacer para lograrlo (Estrategias). En este artículo se ofrece una breve descripción de la evolución histórica de la planeación estratégica en el mundo, que incluye algunas definiciones de autores foráneos y la posterior conceptualización del término. De igual modo, se expone la concepción tradicional de planeación y la estratégica, su importancia, se reflexiona sobre algunas investigaciones realizadas relacionada con la temática y, por último, se describen los pasos metodológicos para su confección, con lo cual el sistema organizacional lograría grandes avances.

Palabras clave: planeación estratégica; metodología de la planificación estratégica.

\section{Abstract}

Strategic Planning serves to determine who we are (Mission) where we want to go (Vision) and what we must do to achieve it (Strategies). This article provides a brief description of the historical evolution of strategic planning in the world, which includes some definitions of foreign authors and the subsequent conceptualization of the term. In the same way, the traditional conception of planning and the strategic one is exposed, its importance, is reflected on some investigations carried out related to the thematic and, finally, the methodological steps for its preparation are described, with which the organizational system would achieve great Advances.

Keywords: strategic planning; strategic planning methodology.

\section{Resumo}

Planejamento Estratégico é usado para determinar que somos (Missão), onde queremos ir (Vision) e que devemos fazer para alcançá-lo (Estratégias). Este artigo fornece uma visão geral da evolução histórica do planejamento estratégico no mundo, incluindo algumas definições de autores estrangeiros e posterior conceptualização do termo. Da mesma forma, o conceito tradicional de planejamento e estratégica exposta, a sua importância, refletimos sobre alguma pesquisa conduzida relacionado ao assunto e, finalmente, os passos metodológicos para torná-los são descritos, em que o sistema organizacional iria realizar grandes progresso.

Palavras chave: planejamento estratégico; metodologia de planejamento estratégico. 


\section{Introducción}

Cada día el mundo se torna más inestable, turbulento e incierto, observándose que el cambio se ha convertido en una regularidad. Fenómenos que no existían hace unas pocas décadas, ya forman parte de los rasgos distintivos del panorama del siglo XXI, y ocasionan impactos negativos sin precedentes en todos los países. Esta realidad, acentuada en las naciones subdesarrolladas, es una permanente amenaza difícil de manejar ya que provoca rupturas, tanto en las relaciones de producción como en la superestructura, que han transformado el orden de las cosas y de las percepciones, cambian el modo de vivir, pensar y actuar de las personas y refuerzan el consumismo, la destrucción del medio ambiente, la agudización de las desigualdades y de la pobreza, entre otros males. Ello obliga a las organizaciones, a reaccionar ante los riesgos y oportunidades con una nueva forma de sustentar su gestión. (Almuiñas Rivero J, Galarza López J. 2012)

El complejo escenario en el que desarrollan el trabajo las organizaciones modernas, incrementa la importancia del empleo de algún sistema de planificación estratégica que sirva de guía para la realización efectiva de su misión, así como para la conformación de una idea más o menos precisa sobre cómo deberán proyectarse en el futuro cercano, cuestión esta última a la que los especialistas suelen denominar visión. (Concepción Báez, C M. 2007).

\section{La planeación estratégica: su evolución histórica y definición}

Etimológicamente la palabra estrategia proviene del griego „,strategia ${ }^{e e}$, que significa "actitudes de un general", acaudillar, que no es más que "planificar la destrucción de los enemigos en razón eficaz de los recursos".

A principios del siglo XX, a nivel internacional se comenzó a utilizar en la literatura científica el concepto planificación, establecido por Taylor y Fayol, según refiere Azocar, quienes buscaban suplantar el empirismo que había en las empresas por un método científico en el que la planificación fungiera como instrumento de dirección. Al respecto, Taylor se dedicó a diseñar los principios de la administración, de cuyo proceso surgieron 4 fundamentales: de planeación, de preparación, de control y, por último, de ejecución. Por su parte, Fayol desarrolló el concepto de administrar, como planear, organizar, dirigir, coordinar y controlar. (Sánchez Jacas, I. 2017). 
La evolución de la planeación estratégica ha sido abordada también por varios teóricos de la teoría administrativa, entre ellos Ansoff. (Márquez EA. 2011). Esta se introdujo por vez primera, a mediados del año 1950, en algunas empresas comerciales, de las cuales las más importantes fueron las que principalmente desarrollaron sistemas de planeación estratégica formal o, como también se les denominó, sistemas de planeación a largo plazo.

Así mismo otros científicos han dado su definición; entre los más significativos estuvieron Sócrates, en la Grecia antigua, y Von Neuman y Morgenstern, con su obra La Teoría del Juego, que abarca el contexto de los negocios. Luego, en el siglo XX, en 1954, Peter Drucker ofreció su definición, y en la década de los 60' se comenzó a emplear el término "planeación a largo plazo". (Sánchez Jacas, I. 2017).

Sobre la base de las consideraciones anteriores la planificación, aunque tuvo su origen en un pasado remoto, como actividad reconocible, es un producto del siglo XX. Su área de competencia genética fue en ambos casos la económica pero, desde allí, se abrió camino hacia todos los demás sectores. Estas circunstancias no fueron insignificantes pues han tenido influencias en los diversos modelos de gestión de planificación, que aún siguen vigentes. (Álvarez A. 2012), (Sánchez Blanco C. 2011).

La historia de la planificación, en general, muestra que ella siempre ha tenido como centro la preocupación por el cambio. Del mismo modo, por ser una herramienta develada para la gestión de procesos, su propensión es la de organizarlos de manera tal que sea posible concretar posible concretar múltiples fines. (Clímaco Cañarte, J. 2012).

El referente de base ha estado en la idea de que el conocimiento, el saber, permite no solo conocer sino también dirigir. Ha sido un reto a la sensatez lógica, al entender que la razón es capaz de discernir qué es lo mejor en una situación determinada y qué no. (Vidal E. 2004), (Clímaco Cañarte, J. 2012).

También es un reto a la voluntad de los actores, pues se asevera que es posible, a través de ella, dirigir los procesos sociales. "En última instancia la planificación se preocupa por el control sobre el futuro. Planeamos en un intento de asegurar que el futuro resulte de tal forma que se aproxime a la manera como lo deseamos, a lo cual Matus agrega la idea de que ella implica un acto de voluntad cuando señala“[...] la planificación, como cuerpo de teoría general, puede 
aplicarse a cualquier actividad humana donde es necesario un esfuerzo para alcanzar un objetivo. (Clímaco Cañarte, J. 2012).

\section{Concepción tradicional de planeación.}

Dentro de la concepción tradicional del concepto de planeación se ponía principal énfasis en lo que podía hacerse, previendo las condiciones en las que debería desarrollarse el futuro de acuerdo a las previsiones hechas, estableciendo con precisión lo que se estaba obligado a hacer. Esta concepción se refería únicamente a las acciones o medidas que podrían ser empleadas en el futuro, fijando el curso concreto de la acción a seguir "estableciendo los principios que habrán de orientarlo, la secuencia de operaciones para realizarlo y las determinaciones de tiempos y de números necesarias para su realización" imprimiéndose su carácter de no flexibilización. La planeación tradicional, por lo general, se realizaba a través de la elaboración de planes en los cuales se suponían que los objetivos finales era rígidos, las variables permanecían estables.

Sin embargo, "la rapidez de los cambios y el fenómeno de la globalidad introducen en las organizaciones la conciencia de la incertidumbre, al mismo tiempo la acción pública se desenvuelve en sociedades complejas, diversas y multiculturales", motivo por el cual la administración y planeación tradicional se vieron impedidas para dar res puesta satisfactoria al acumulo de problemas derivados de la multiplicidad de interrelaciones en el mundo. (Méndez Ramírez J, Becerril Sánchez T. 2005).

Las organizaciones públicas dieron un giro a la concepción de la planeación, al pasar del conocimiento y empleo de acciones que indicaban los caminos adecuados, la reducción de tiempos y la obtención de mayores beneficios, a la adopción de una visión más holística de la realidad a fin de que la planeación "ahora estratégica" al tener un mayor vínculo con su entorno pudiese responder de mejor manera a los requerimientos sociales. (Méndez Ramírez J, Becerril Sánchez T. 2005).

\section{Planeación estratégica}

El concepto de planeación estratégica se aleja cada vez más de la planeación tradicional dado que en ella se plantean los objetivos finales en forma más flexible, de tal manera que puedan ir evolucionando con el tiempo; al mismo tiempo, las variables del contexto son cambiantes e 
inestables, permitiendo con ello que las organizaciones tengan la capacidad de adaptación necesaria para adecuar se a las distintas rea li da des o con tex tos sociales. La planeación estratégica observa las posibles alternativas de acción en el futuro, y al escoger dichas alternativas, éstas se convierten en la base para tomar decisiones presentes. (Méndez Ramírez J, Becerril Sánchez T. 2005).

De tal modo la planeación estratégica no es un conjunto de técnicas específicas o de métodos y procedimientos inflexibles para elaborar un plan. Para generar la planeación estratégica es importante cuestionarnos lo siguiente: ¿Dónde estamos? ¿A dónde vamos? ¿A dónde deberíamos de ir?

Igualmente la planeación estratégica incluye eventos factibles pero, no obstante es preciso considerar eventos contingentes que pueden llegar a ocurrir. La planeación no constituye una respuesta informal o fortuita a una crisis. La planeación formal es un proceso para la toma de decisiones, los pasos importantes que se siguen durante ella se asemejan a los pasos básicos para la toma de decisiones. La planeación implica directamente problemas u objetos reales, no simples especulaciones. (Cendejas Valdés J. 2014).

La planificación estratégica se operacionaliza a través de un proceso, cuyo principal resultado es la Estrategia institucional. Mantener altos rendimientos en la gestión de dicho proceso exige desarrollarlo con calidad, y por tanto se necesitan acciones evaluativas, que propicien su mejoramiento continuo. (Almuiñas Rivero J, Galarza López J. 2012).

Los planificadores deben ser competentes en el manejo técnico de las herramientas de su tarea; contar con sensibilidad para equilibrar los excesos a que en ocasiones los lleva el mercado. Así, la planificación en el ámbito social se comenzó a desarrollar asumiendo la imposibilidad de coordinar todos los procesos de planificación en una sociedad abierta. Sin embargo, al mismo tiempo hacía explícita la necesidad de ser aplicada en la reducción de las consecuencias contrarias al sentido de justicia y a la funcionalidad del modelo económico de la racionalidad del mercado, así como para estructurar un marco de referencia para la estabilidad y la competitividad económica. (Almuiñas Rivero J, Galarza López J. 2012). 
Es evidente entonces que el concepto estratégico no es nuevo ya que siempre ha estado en la mente de los líderes, hombres de negocios y gerentes lo nuevo es el manejo sistémico de la planeación estratégica. (Clímaco Cañarte, J. 2012).

\section{Importancia de la planeación estratégica.}

Se podría afirmar que la planeación estratégica resulta ser clave en la organización de la administración tanto privada como pública, por ser considerada una "herramienta que permite a las organizaciones alcanzar la visión de éxito gracias a la aplicación de un método sistemático de definición de acciones, método que, a su vez, auxilia en la generación de un consenso sobre la participación, el compromiso y la priorización de las mis más en el proceso de la gestión. Estratégica. (Méndez Ramírez J, Becerril Sánchez T. 2005).

Según Clímaco Cañarte (2012), en su investigación expresa que el proceso de planificación estratégica universitaria es un instrumento de gestión social que sintetiza los principios básicos de la formulación de políticas sociales, como la participación ciudadana de los grupos sociales involucrados en políticas; la generación de consensos a través de la negociación y la generación de políticas a largo plazo para el logro del desarrollo endógeno. Aún le queda camino por recorrer para el logro de una mayor profundización en la temática, de manera que ella pueda ser aplicada al proceso formativo universitario. Otro estudio realizado por (Almuiñas Rivero J, Galarza López J. 2012), en el escenario universitario los resultados del análisis ponen en evidencia la presencia de diferentes tipos de problemas: conceptuales, metodológicos y técnicos, organizativos, culturales, entre otros, profundizando en la necesidad de realizar este tipo de evaluación en las universidades, con vistas a detectar a tiempo señales de aviso, que indiquen la presencia de insuficiencias y nuevas fortalezas existentes no descubiertas, para mantener en alto la calidad de dicho proceso y por tanto, mejorar la gestión institucional .

Jiménez Hernández C N (2006), realizó un ejercicio de planeación estratégica contando con la participación de representantes de la comunidad biotecnológica colombiana, quienes conforman el Consejo editorial de la Revista Colombiana de Biotecnología - RCB, única publicación seriada especializada en esta temática en el país. Con este ejercicio de planeación estratégica se observó que para todo tipo de organizaciones es indispensable tener claridad sobre el rumbo que se sigue 
y sobre las metas perseguidas, con el fin de generar resultados e impactos tangibles evitando estar a la deriva, duplicar esfuerzos y malgastar recursos.

Finalmente, se hace necesario que las organizaciones se preparen para los continuos cambios con flexibilidad tanto en sus funciones como en sus estructuras y procurando que tales cambios se produzcan como consecuencia de la generación y asimilación de conocimiento por parte de sus miembros, o que dicho conocimiento constituya la base para la anticipación proactiva.

Después de una exhaustiva revisión bibliográfica respecto a una metodología para la confección de planeación estratégica, en la investigación realizada por Sánchez Jacas I (2017) expone la siguiente:

\section{Metodología para su confección}

- Introducción: donde se exprese de forma breve y general qué es la planeación estratégica, qué importancia tiene su confección para los directivos, qué plantea sucintamente la situación actual, dónde estamos y dónde queremos llegar; aspectos que se profundizarán en la confección de la misión y visión más adelante. No debe ser mayor de una cuartilla.

- Objetivo(s): de la planificación, donde se señale el sistema o subsistema en que esta es diseñada y el período que abarcará. La mayoría de las bibliografías plantean un mínimo de 3 años.

- Métodos: se expresarán de forma detallada las etapas concebidas para la realización de la PE, quiénes fueron los participantes, qué técnicas se utilizaron para algunos acápites, cómo es la identificación y priorización de problemas.

- Caracterización de la institución - denominada también identidad de la institución - (situación actual): se expondrá la ubicación geográfica, que incluya centros históricos, industriales, de servicios o de producción importantes; la estructura de la institución; el capital humano; los materiales de que disponen y, si es posible, los financieros; con qué cuenta la institución para brindar servicios a la población o impartir docencia. En otras publicaciones se resume este aspecto en: antigüedad de la dirección, tamaño, campo de actividad, forma de dirección, ámbito geográfico y forma jurídica.

- Misión: la razón de ser de la institución. 
- Visión: debe ser del sistema organizacional.

- Identificación y priorización de los problemas

- Problema principal:

- Valores

- Escenarios

- Campos de fuerzas: fortalezas, debilidades, oportunidades y amenazas.

- Factores de éxito

- Objetivos estratégico

Plan de acción

- Evaluación.

\section{Referencias bibliográficas}

Almuiñas Rivero J, Galarza López J. (2012). El proceso de planificación estratégica en las universidades: desencuentros y retos para el mejoramiento de su calidad. Revista GUAL, Florianópolis, 5(2), p. 72-97

Álvarez A. (2012). La magia del planner. Madrid: Esic Editorial.

Concepción Báez, Carlos M. (2007). La planificación estratégica como herramienta. ACIMED, 16(2) Recuperado de http://scielo.sld.cu/scielo.php?script=sci_arttext\&pid=S1024$94352007000800014 \& \operatorname{lng}=$ es\&tlng=es.

Clímaco Cañarte, J. (2012). Antecedentes del proceso de planeación estratégica como fundamentos para el logro de un desarrollo endógeno sustentable desde la universidad. Humanidades Médicas, 12(3), 464-486. Recuperado de http://scielo.sld.cu/scielo.php?script=sci_arttext\&pid=S1727$81202012000300008 \& \operatorname{lng}=\mathrm{es} \& \operatorname{lng}=\mathrm{es}$

Cendejas Valdés J. (2014). Planeación estratégica. Recuperado de http://www.eumed.net/tesisdoctorales/2014/jlcv/planeacion-estrategica.htm 
Jiménez Hernández C N, Castellanos Domínguez O F (2006). Herramientas de planeación estratégica para el direccionamiento de procesos de divulgación científica. Congreso Iberoamericano de Ciencias Tecnología y Sociedad. Recuperado de www.oei.es/historico/memoriasctsi/mesa1/m01p27.pdf

Márquez EA. (2011) Estudio y análisis de la Matriz de Igor Ansoff. Recuperado de: https://es.slideshare.net/adelismarquez/igor-ansoff-10132220

Méndez Ramírez J, Becerril Sánchez T. (2005). Planeación estratégica: técnica gerencial aplicada en la administración pública. Quivera, 7(2), pp. 160-178 Universidad Autónoma del Estado de México Toluca, México

Sánchez Blanco C. (2011). Planificación estratégica. Madrid: Editorial Universitas

Sánchez Jacas, Isolina. (2017). La planeación estratégica en el Sistema de Salud cubano.

MEDISAN, 21(5), 635-641. Recuperado de

http://scielo.sld.cu/scielo.php?script=sci_arttext\&pid=S1029

$30192017000500019 \& \operatorname{lng}=\mathrm{es} \& \operatorname{tlng}=\mathrm{es}$

Vidal E. (2004). Diagnóstico organizacional. Bogotá: Ecoe Ediciones. 